TEMA:

APRENDIZAJE ORGANIZACIONAL COMO UNA ESTRATEGIA

COMPETITIVA PARA EL SECTOR HOTELERO DE BOGOTÁ - ZONA CHILE

TUTOR:

Mauricio Novoa

PRESENTA:

Paula Andrea Rodríguez García

9no semestre

Bogotá D.C, Colombia

12 de Marzo del 2019 


\section{EL APRENDIZAJE \\ ORGANIZACIONAL COMO \\ UNA ESTRATEGIA \\ COMPETITIVA PARA EL \\ SECTOR HOTELERO DE \\ BOGOTÁ - ZONA CHILE'}

PAULA ANDREA RODRÍGUEZ GARCÍA ${ }^{2}$

\section{RESUMEN}

El aprendizaje organizacional en la actualidad es considerado como una de las principales herramientas estratégicas para incrementar el rendimiento en los colaboradores y así mismo, crear una ventaja competitividad para las compañías en general y para las empresas hoteleras en particular. Además, el sector hotelero para maximizar sus recursos y garantizar su constante renovación se conforman de los sistemas de información como instrumento de apoyo.

Este artículo, de carácter exploratorio tiene como objetivo dar a conocer los componentes estratégicos, medidas de desempeño y procesos formales de aprendizaje que se implementa en el Sector Hotelero de Bogotá - Zona Chile.

\footnotetext{
${ }^{1}$ Artículo resultado de la investigación sobre el Aprendizaje Organizacional desarrollado en el sector hotelero Zona Chile bajo la tutoría de los docentes Mauricio Novoa y Jairo Contreras.

${ }^{2}$ Estudiante de $9^{\circ}$ Semestre de Administración de Empresas, Universidad Santo Tomás.
}

\section{Palabras Claves:}

Aprendizaje Organizacional, Sector Hotelero, Ventaja Competitiva.

\section{ABSTRACT}

Organizational learning is currently regarded as one of the main strategic tools to increase performance of employees and also create an advantage competitiveness for companies in general and for the hotel companies in particular. In addition, the hotel sector to maximize its resources and ensure its constant renewal will be adjusted to the information systems as a support instrument.

This article, which is of an exploratory nature, aims to publicize the strategic components, performance measures and formal learning processes that are implemented in the Hotel Sector of Bogotá - Zona Chile.

\section{Key words:}

Organizational learning, hotel industry, competitive advantage.

\section{INTRODUCCIÓN}

Durante los últimos años numerosos autores abundan en considerar al conocimiento como el recurso más importante que posee una organización para conseguir y mantener una ventaja competitiva (Drucker, 1993). Por ejemplo, Fiol y Lyles (1985) definen el aprendizaje como "el proceso de mejorar las 
acciones mediante un conocimiento y una comprensión mejores" (p 803). No obstante, las organizaciones aprenden, en el sentido propuesto por Leacitt y March (1988), cuando "codifican las inferencias de la historia en rutinas que guían la conducta" ( $p$ 319). Huber (1989) sugiere que: una organización ha aprendido si cualquiera de sus componentes ha adquirido información y ha hecho que dicha información esté disponible para su empleo, ya sea de parte de otros componentes o de él mismo, en nombre de la organización (p3).

Es decir, el aprendizaje organizacional ha adquirido una relevancia significativa en las organizaciones cuyo objetivo busque fomentar el aprendizaje en el ámbito individual, grupal y organizativo donde se intercambie conocimiento e información en todos los niveles jerárquicos de la compañía. No obstante, algunos autores critican el aprendizaje organizacional debido a que afirman que gran parte de él, tal vez incluso la mayor parte, está al servicio de la estabilidad, más que del cambio. Conforme a este punto de vista, las organizaciones aprenden a preservar el statu quo y el aprendizaje de este tipo es el enemigo del cambio y de la reforma organizacionales. (Argyris C., 2001)

Por otro lado, cabe resaltar que de acuerdo a (Es.investinbogota.org, 2016) Bogotá es considerado como el epicentro del turismo de negocios o participación de eventos corporativos del total de viajeros, ubicándola en el primer lugar de preferencia como puerta de entrada del turismo en Colombia, por encima de ciudades como Cartagena, Cali y Medellín. Además, en los últimos 10 años Bogotá concentró el $37 \%$ de la inversión hotelera en el país, donde el Instituto Distrital de Turismo (IDT) concluyó que en el transcurso de estos últimos 6 años se evidenció un incremento en el número de habitaciones en Bogotá desde 11.000 hasta 17.030

El sector hotelero es un sector dinámico, con un alto grado de atomización y que necesita aprender de sus clientes para conseguir fidelizarlos (Hill y Jones, 2005; Rodríguez Antón y Alonso Almeida, 2008). Partiendo de esta hipótesis, el contenido de este artículo tiene como finalidad discutir la gestión y aplicación del aprendizaje organizacional en el sector hotelero de Bogotá, específicamente en la Zona Chile a partir de una investigación exploratoria y revisión bibliográfica, además de surgir el siguiente interrogante: ¿Qué tipo de estrategias competitivas se implementan en el Sector Hotelero de Bogotá - Zona Chile?

\section{MARCO TEÓRICO}

\section{PETER SENGE:}

Peter Senge, pionero en desarrollo de aprendizaje organizacional en las empresas estadounidenses, "es claro que no hay fórmulas mágicas para construir aprendizaje organizacional... El aprendizaje debe ser continuo y crear ambientes de trabajo que inspiren producir resultados $\mathrm{y}$ generen diversión al hacerlo". En sus diferentes entrevistas y análisis en empresas como Intel 
y Nike, entre otras, reveló el desarrollo del aprendizaje organizacional, y el valor que tiene este activo intangible en el crecimiento de la compañía. Sus conclusiones, derivadas del estudio de 100 casos en aprendizaje organizacional, lo plasmó en su libro La quinta disciplina, en el cual revela 8 estrategias para incentivar este tipo de aprendizaje. Entre ellas están la creación de un pensamiento sistémico, dominio personal y la creación de modelos mentales que permitan llevar a visiones compartidas y a un aprendizaje compartido. Esto estimula los resultados así como mejores desempeños cimentados en acciones concretas que conllevan la preparación de actividades de entrenamiento capaces de dar nuevas formas y nuevos contenidos a la organización para perdurar. (Gracia, 2009)

Con un enfoque de pensamiento sistémico, Senge alienta a ampliar el campo de observación de las organizaciones para comprender complejidades e interacciones. Mientras que la visión tradicional de las empresas era la de fenómenos parcelados independientes entre sí, el enfoque sistémico propone pensar en totalidades y hacer conexiones, buscando puntos de mejora o apalancamientos que restablezcan el equilibrio del sistema y le permitan volverse abierto al aprendizaje y la auto superación.

El pensamiento sistémico es el eje central del cual se derivan organizaciones inteligentes como lo propone Senge. Oconnor nos plantea que la resistencia al cambio es una caracterìstica inevitable a encontrar en todo sistema que goce de cierta estabilidad, por tanto no puede haber estabilidad sin resistencia. Y al anunciar como introducciòn la importancia del aprendizaje quiero tomar dos de las disciplinas que refiere Senge entre sus cinco, una es los modelos mentales, los cuales define como la apertura necesaria para superar las limitaciones en nuestra forma de ver el mundo, y la otra es el dominio personal, el cual define como la disciplina que nos permitirà aclarar $\mathrm{y}$ ahondar continuamente nuestra visiòn personal, concentrando energìas y desarrollando paciencia que permitiràn ver la realidad objetivamente .

Por tanto uno de los puntos de partida para iniciar procesos de cambio creo debe ser este ènfasis en lo personal, el cual debe iniciarse desde las esferas de liderazgo màs altas de la empresa, de manera que estos puedan constituirse en modelos a imitar en el proceso de generar pensamiento sistèmico en los miembros del sistema. De igual manera lo sugiere Oconnor al decir, que mientras màs alto es el nivel de control de la parte en la que se fectuarà el cambio, màs se extienden y ramifican sus efectos.

Senge, nos plantea que son las estructuras las que generan determinadas conductas, tambièn partiendo de cada una de las conductas se genera la estructura y creo es éste el punto de partida para generar organizaciones inteligentes, o al menos esta es la aproximaciòn que le veo a la puesta en práctica (Gracia, 2009)

\section{"Las organizaciones inteligentes son} posibles porque en el fondo todos somos aprendices. Nadie tiene que enseñar a un niño a aprender. En rigor, nadie tiene que enseñar nada a un niño. Los niños son intrinsecamente inquisitivos, aprendices hábiles que aprenden a caminar, hablary apañárselas por su cuenta. Las organizaciones inteligentes son posibles porque aprender no sólo forma parte de 
nuestra naturaleza sino que amamos

aprender". SENGE, P. (1992:2)

\section{HIROTAKA TAKEUCHI E IKUJIRO} NONAKA :

Como menciona (Aguilera, 2017) el Modelo para la Gestión del conocimiento organizacional de Nonaka y Takeuchi hacen referencia a dos dimensiones importantes del conocimiento para poder llegar a entender la naturaleza del conocimiento organizacional entre las cuales se destaca la dimensiòn ontológica y epistemológica.

Dimensión ontológica del conocimiento: Se considera que la creación de conocimiento individual se encuentra centrada en diferentes categorías como grupales, individual,organizacional e interorganizacional.

Estos autores se centran en que el conocimiento es elaborado netamente por los individuos y la sociedad, por lo cual la organización está sujeta a contar con individuos ya que sin ellos no sería posible su creaci

Dimensión epistemológica del conocimiento: En esta dimensión se basa primordialmente los diferentes procesos que se utilizan para comunicar el conocimiento (Tácito y explícito):

- Tácito: Es considerado un conocimiento personal, difícil de trasmitir originándose en los valores, los ideales y las experiencias de cada persona

- EXPLÍCITO: Las personas lo expresan con mayor facilidad por medio de un lenguaje formal basándose en palabras, transmitiendo las y comunicándose con mayor facilidad.

Proceso de conversión de conocimiento según Nonaka y Takeuchi:

En este proceso se empieza a dar la coacción entre el conocimiento tácito y el explícito explicados anteriormente los cuales son llevados a cabo por los individuos encontrándose ais 4 etapas importantes para llevar a cabo estos procesos:

- Socialización: Este conocimiento es compartido por medio de las experiencias que han tenido los individuos desarrollandolo por medio de la práctica y la imitación implantando en modelos mentales para que sea más fácil de compartir con su grupo de trabajo o con las personas que los rodean generando el "conocimiento armonizado".

- Exteriorización: En esta etapa los conocimientos que eran tácitos son convertidos en explícito permitiendo que estos sean más comprensible y entendido por todos los miembros de la organización permitiendo que estos interactúen durante la comprensión y la adquisición del conocimiento.

- Combinación: En este punto los conocimientos que se están impartiendo son más formales para que cada miembro de la organización acceda a él con más facilidad, se comienza a difundir mediante conferencias y presentaciones permitiéndoles ser accesible para todos. 
- Interiorización: Se puede evidenciar los miembros de la organización comienzan a poner en práctica esos conocimientos volviendo de lo explícito a lo tácito analizando por medio de esta las experiencias que se adquirieron durante el proceso $\mathrm{y}$ poniendolos en practica por medio de modelos mentales y talleres relacionados con el trabajo que ellos realizan dentro de la organización.

La espiral de creación de conocimiento dentro de las organizaciones:

Las organizaciones son las encargadas de proveer los recursos facilitadores para poder llevar a cabo actividades grupales permitiendo la acumulacion del conocimiento ya sea indivial o grupal esto está restringido o condicionado por 5 requerimientos que pueden llegar a facilitar este espiral de conocimiento:

- Intención: Necesario para alcanzar las metas que la organización se proponen,por lo cual la empresa es la encargada de apoyar ese compromiso por la creación de conocimiento basándose en actividades integrando la teoría del pensamiento estratégico implantado en la visión.

- Autonomía: Como la organización facilita o posibilita que sus miembros actúan de manera autónoma permitiendo que se generen nuevos conocimientos por medio de ideas y la generación de nuevas oportunidades.

- Caos creativo: Estimular que los integrantes de la organización interactúen en un ambiente en donde enfrentan diferentes retos, rutinas y generar estrategias para afrontar tiempos de crisis por medio de la implementación de conocimientos que sean necesarios para estos casos.

- Redundancia: Esto se genera por medio de la rotación de personal esto generada por medio de una estrategia se da en diferentes áreas en donde se tengan diferencias ya sea por tecnología o funciones,por otro lado esto se puede evidenciar por medio de las comunidades de conocimiento combinando la informa de manera distinta para aumentar la variación y permitiendo accedan más rápido a la información.

Según estos dos importantes autores la fases que componen la gestión del conocimiento son cinco:

- Conocimiento tácito.

- Conocimiento conceptual.

- Conocimiento armonizado.

- Conocimiento sistémico.

- Conocimiento operacional.

\section{TIPO DE INVESTIGACIÓN}

En la presente se desarrolló una investigación descriptiva tipo concluyente derivada del paradigma positivista, donde las mismas son objetivas, este tipo de investigación tuvo como objetivo verificar las hipótesis planteadas y encontrar una relación, además se caracterizó por tener unas necesidades definidas; el proceso de investigación fue 
formal y estructurado y se tomaron muestra grandes para así mismo tener mayor precisión, a las muestras se les aplicaron técnicas de análisis cuantitativo.

\section{ENFOQUE METODOLÓGICO}

En la investigación se aplicó un enfoque metodológico cuantitativo, el cual tiene como objetivo cuantificar los datos que son obtenidos de una muestra de la población, los cuales fueron los hoteles de la ciudad de Bogotá - Zona Chile clasificados por la Asociación Hotelera Colombiana (COTELCO); luego se analizaron los datos por medio de SPSS, el cual es un software estadístico que permite obtener mejores resultados e interpretaciones de los datos recolectados.

\section{TÉCNICAS CUANTITATIVAS E INSTRUMENTOS DE RECOLECCIÓN DE INFORMACIÓN}

En el presente estudio de acuerdo al enfoque metodológico cuantitativo de tipo descriptivo, se implementó la técnica de encuesta por internet y personal, por medio del instrumento cuestionario semi estructurado con formato convencional de tipo descriptivo, por medio de este se buscó lograr los resultados de acuerdo a las preguntas implementadas en dicho instrumento, y conocer las características, componentes estratégicos y sistemas de información que permiten fomentar y facilitar el aprendizaje organizacional y personal en estas cadenas hoteleras de la ciudad de Bogotá. Se tuvo en cuenta dos medios para la difusión del cuestionario para la recolección de información, uno físico y otro digital.

\section{INSTRUMENTOS EMPLEADOS}

El tipo de instrumento fue un cuestionario de formato convencional, y semi-estructurada, ya que posee preguntas abiertas; este enfocó sus preguntas a poder identificar los componentes y características más importantes de la estructura organizacional de las empresas hoteleras de Bogotá, posterior a esto las preguntas se enfocaron en determinar qué sistemas de información se cuenta al interior de la organización para proporcionar datos sobre el desempeño de los colaboradores, y a su vez que prácticas e incentivos implementan para promocionar el aprendizaje organizacional. El instrumento está conformado por preguntas cerradas de respuesta múltiple, cerradas dicotómicas, preguntas abiertas, abierta de clarificación, escala de extremadamente importante a nada importante según Likert.

\section{GRUPO OBJETIVO}

Esta investigación va dirigida a los gerentes de Talento Humano de los Hoteles Hilton, Bs Rosales, BH Tempo, Scala 68, Vilar América, Suit Lugano Imperial SAS de la ciudad de Bogotá - Zona Chile clasificados por COTELCO. 


\section{RESULTADOS}

1.¿Cuál es la característica más importante de la estructura organizacional?

Tabla 1:

Características importantes de la estructura organizacional

\begin{tabular}{|c|c|}
\hline CARACTERÍSTICAS & $\%$ \\
\hline Democrática & $33 \%$ \\
\hline Plana & $17 \%$ \\
\hline Centralizada & $33 \%$ \\
\hline $\begin{array}{c}\text { Democrática / } \\
\text { Centralizada }\end{array}$ & $17 \%$ \\
\hline TOTAL & $100 \%$ \\
\hline
\end{tabular}

Fuente: Elaboración propia

Gráfico 1: Características importantes de la estructura organizacional

¿Cuál es la características más importante de la estructura organizacional?

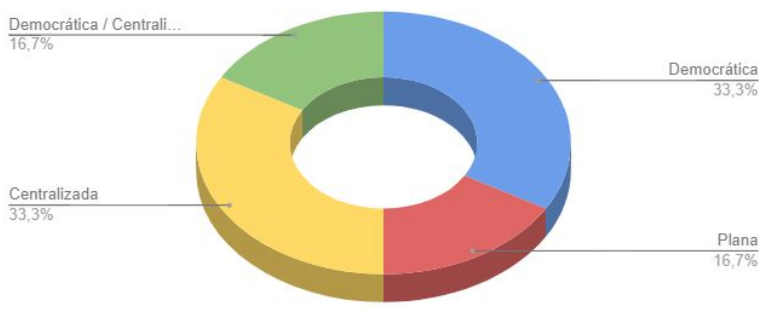

Fuente: Elaboración propia
2.¿El componente estratégico en la organización contempla el Aprendizaje de las personas?

Tabla 2:

Componentes estratégicos frente al Aprendizaje de las personas

\begin{tabular}{|c|c|}
\hline APRENDIZAJE & $\%$ \\
\hline PERSONAS & \\
\hline SI & $\mathbf{1 0 0} \%$ \\
\hline NO & $\mathbf{0 \%}$ \\
\hline TOTAL & $\mathbf{1 0 0} \%$ \\
\hline
\end{tabular}

Fuente: Elaboración propia

Gráfico 1: Componentes estratégicos frente al Aprendizaje de las personas

\section{El componente estratégico en la organización contempla el Aprendizaje de las Personas?}

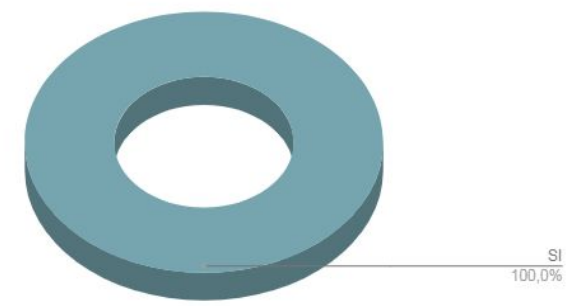

Fuente: Elaboración propia

¿El componente estratégico en la organización contempla el Aprendizaje de la Organización? 
Tabla 2.1:

Componentes estratégicos frente al Aprendizaje de la Organización

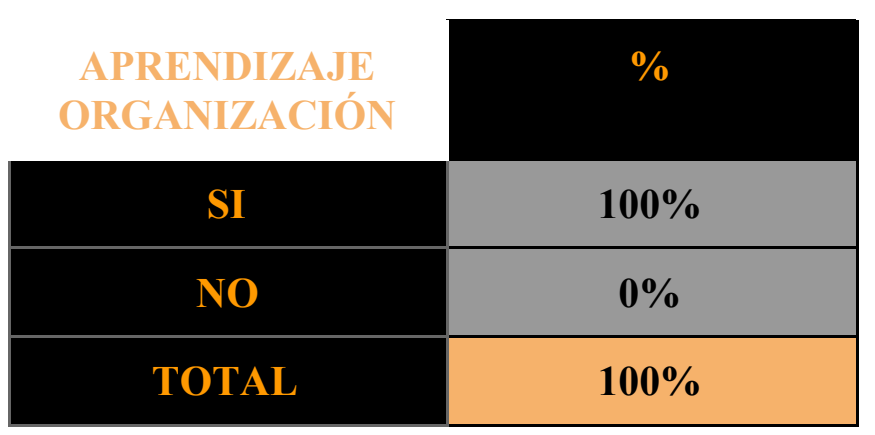

Fuente: Elaboración propia

Gráfico 2.1: Componentes estratégicos frente al Aprendizaje de la Organización

¿El componente estratégico en la organización contempla el Aprendizaje de la Organización?

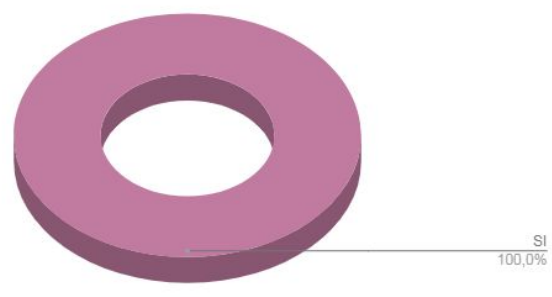

Fuente: Elaboración propia

2.1.¿En qué componentes estratégicos se evidencia el aprendizaje?

\section{Tabla 2.2:}

Componentes estratégicos evidenciados en el Aprendizaje

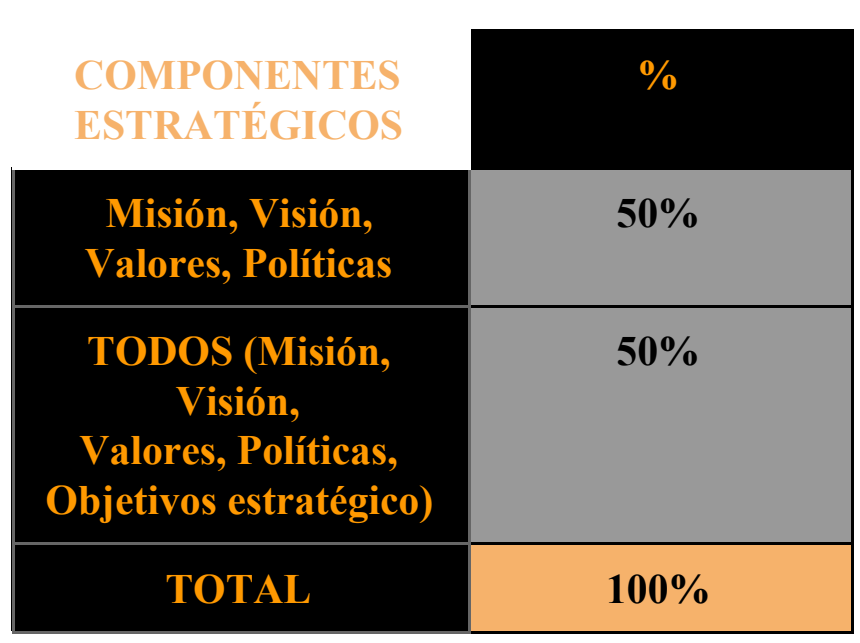

Fuente: Elaboración propia

\section{Gráfico 2.2: Componentes estratégicos evidenciados en el Aprendizaje}

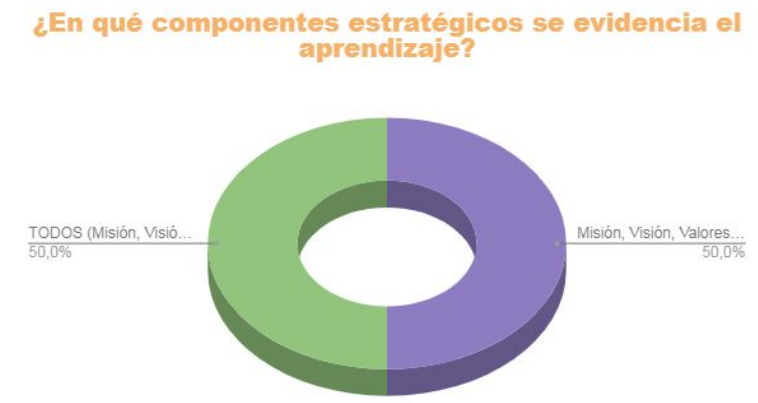

Fuente: Elaboración propia

3. ¿La organización cuenta con medidas de desempeño organizacional? 
Tabla 3:

Medidas de desempeño organizacional

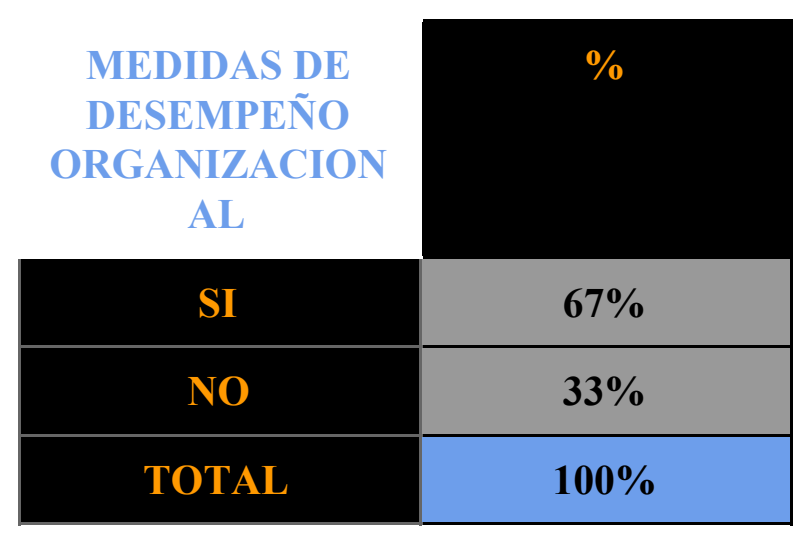

Fuente: Elaboración propia

Gráfico 3: Medidas de desempeño organizacional

¿La organización cuenta con medidas de desempeño organizacional?

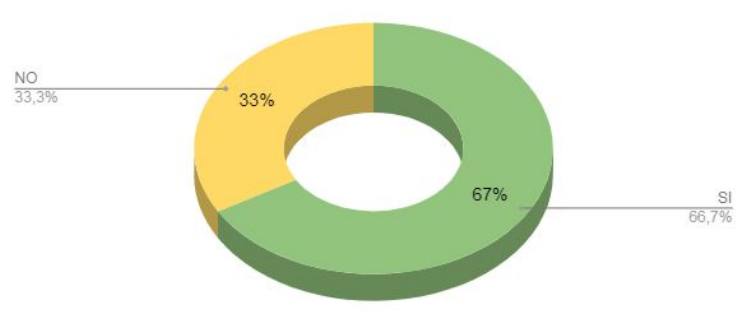

Fuente: Elaboración propia

¿Cuáles son las medidas de desempeño con que cuenta la organización?

Tabla 3.1:

Clases de medidas de desempeño organizacional
MEDIDAS DE

DESEMPEÑO

ORGANIZACIONAL

Evaluación de desempeño

(Cada 6 meses 0 año)

Plan de desarrollo,

Evaluación de desempeño

y Cultura del desempeño

TOTAL

$100 \%$

Fuente: Elaboración propia

Gráfico 3.1: Clases de medidas de desempeño organizacional

¿Cuáles son las medidas de desempeño con que cuenta la organización?

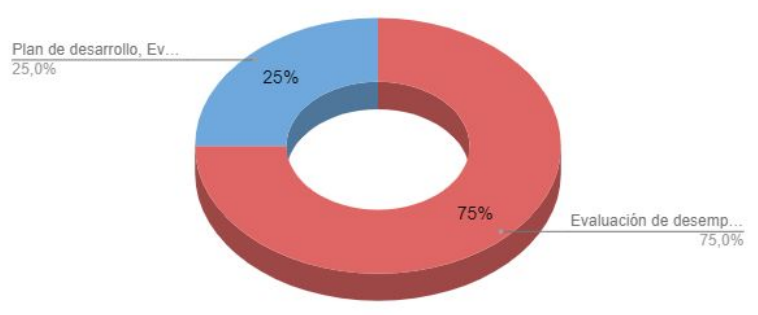

Fuente: Elaboración propia

4. ¿Se tienen sistemas de información que proporcionen datos sobre el desempeño de la organización y de sus componentes?

Tabla 4:

Sistemas de información sobre el desempeño de la organización 


\begin{tabular}{|c|c|}
\hline $\begin{array}{c}\text { SISTEMAS DE } \\
\text { INFORMACIÓN }\end{array}$ & $\%$ \\
\hline SI & $33 \%$ \\
\hline NO & $67 \%$ \\
\hline TOTAL & $100 \%$ \\
\hline
\end{tabular}

Fuente: Elaboración propia

Gráfico 4: Sistemas de información sobre el desempeño de la organización

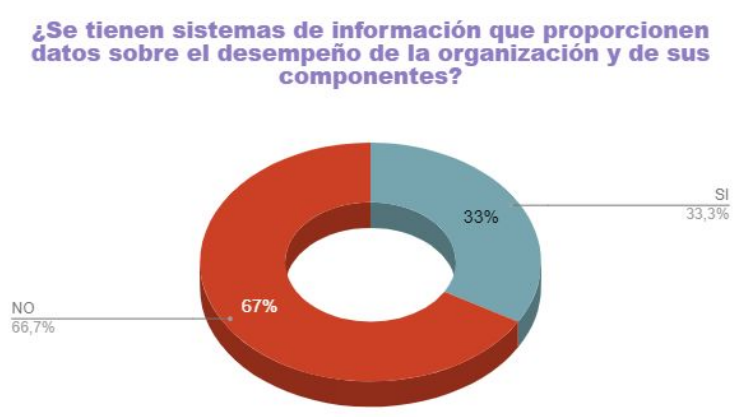

Fuente: Elaboración propia

¿Cuáles son los SI que proporcionen datos sobre el desempeño de la organización y de sus componentes?

Tabla 4.1:

Clases de Sistemas de información sobre el desempeño de la organización

\begin{tabular}{|c|c|}
\hline $\begin{array}{l}\text { SISTEMAS DE } \\
\text { INFORMACIÓN }\end{array}$ & $\%$ \\
\hline $\begin{array}{l}\text { Success Factors } \\
\text { (Herramienta propia } \\
\text { del Hilton para el } \\
\text { Comité Gerencial) }\end{array}$ & $25 \%$ \\
\hline $\begin{array}{l}\text { Evaluación de } \\
\text { desempeño } \\
\text { (Bimensual) }\end{array}$ & $25 \%$ \\
\hline $\begin{array}{l}\text { No se cuenta con } \\
\text { sistema de } \\
\text { retroalimentación, } \\
\text { sin embargo, se } \\
\text { proyecta la } \\
\text { implementación de } \\
\text { un SI }\end{array}$ & $50 \%$ \\
\hline TOTAL & $100 \%$ \\
\hline
\end{tabular}

Fuente: Elaboración propia

Gráfico 4.1: Sistemas de información sobre el desempeño de la organización

¿Cuáles son los SI que proporcionen datos sobre el desempeño de la organización y de sus compone...

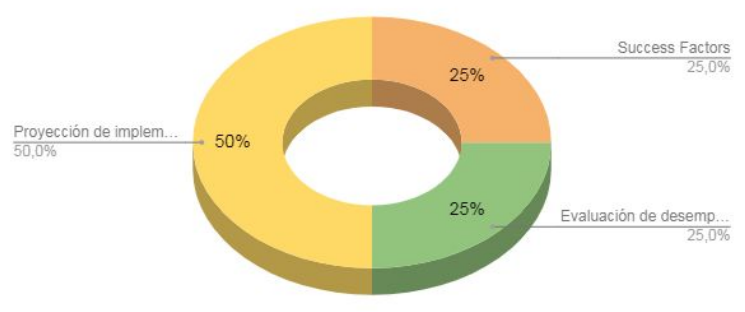

Fuente: Elaboración propia 
4.1. ¿Esos sistemas de información permiten la retroalimentación?

Tabla 4.2:

Sistemas de información que permiten la Retroalimentación

$\left.\begin{array}{|c|c|}\hline \text { SISTEMAS DE } \\ \text { INFORMACIÓN }\end{array}\right)$

Fuente: Elaboración propia

Gráfico 4.2: Sistemas de información que permiten la Retroalimentación

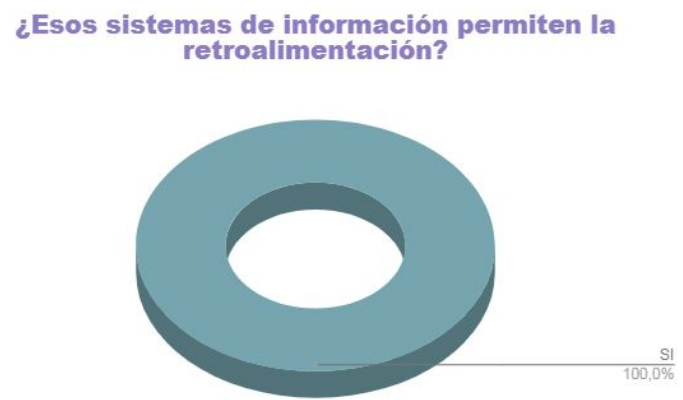

Fuente: Elaboración propia

¿De qué forma esos sistemas de información permiten la retroalimentación?
SISTEMAS DE

$\%$

INFORMACIÓN

\begin{tabular}{|c|c|}
\hline $\begin{array}{c}\text { Revisión y seguimiento } \\
\text { de los planes de } \\
\text { desarrollo y objetivos } \\
\text { (Cada 6 meses) }\end{array}$ & $\mathbf{5 0 \%}$ \\
\hline $\begin{array}{c}\text { Retroalimentación } \\
\text { directa con el } \\
\text { trabajador }\end{array}$ & $\mathbf{5 0 \%}$ \\
\hline TOTAL & $100 \%$ \\
\hline
\end{tabular}

¿De qué forma esos sistemas de información permiten la retroalimentación?

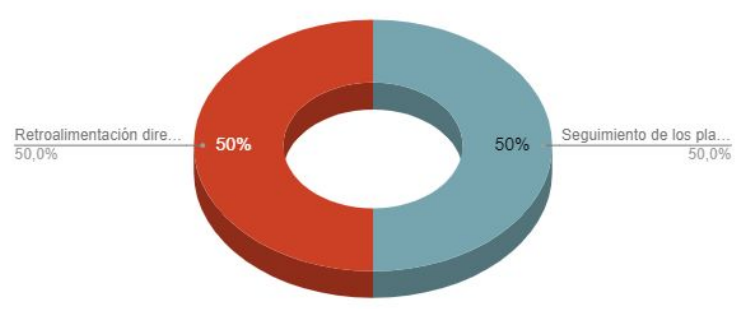

5. ¿Se permite o facilita la investigación experimental?

Tabla 5:

Facilidades en la Investigación experimental

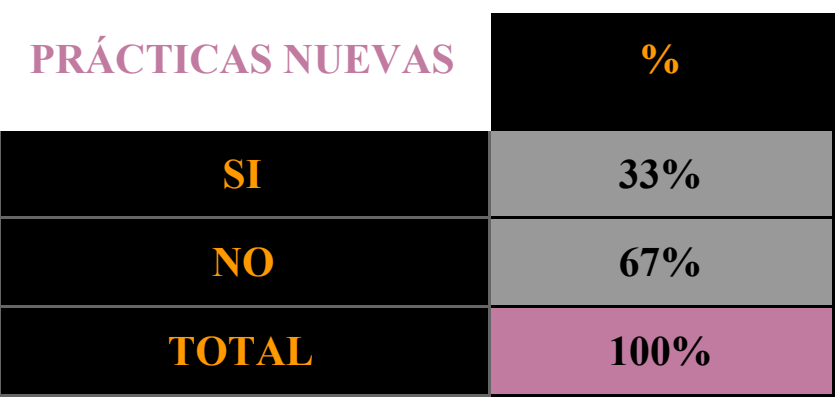

Fuente: Elaboración propia 
Gráfico 5: Facilidades en la Investigación experimental

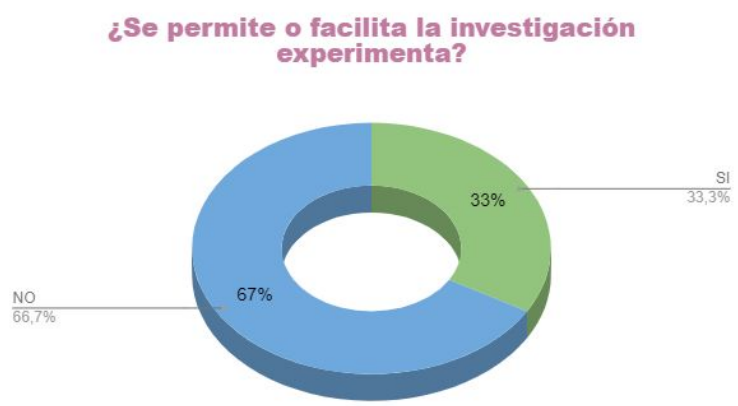

Fuente: Elaboración propia

¿Cómo se realiza la investigación experimental?

Tabla 5.1:

Tipos de facilidades en la Investigación experimental

\begin{tabular}{|c|c|}
\hline PRÁCTICAS NUEVAS & $\%$ \\
\hline $\begin{array}{c}\text { Dirigidas por los jefes de } \\
\text { cada área }\end{array}$ & $\mathbf{5 0 \%}$ \\
\hline $\begin{array}{c}\text { Contratación de } \\
\text { estudiantes del Sena }\end{array}$ & $\mathbf{5 0 \%}$ \\
\hline TOTAL & $\mathbf{1 0 0 \%}$ \\
\hline
\end{tabular}

Fuente: Elaboración propia

¿Cómo se realiza la investigación experimental?

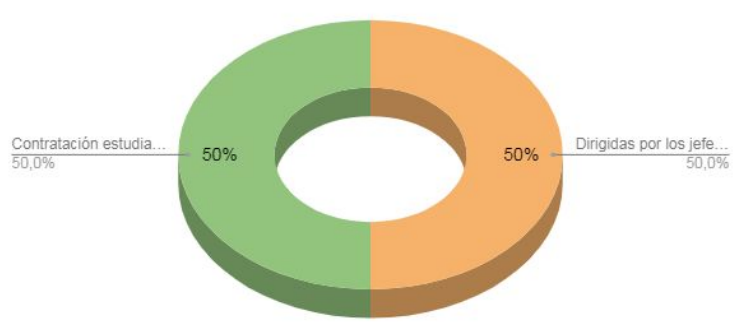

Fuente: Elaboración propia
6. ¿Se cuenta con incentivos para promocionar el aprendizaje organizacional?

Tabla 6:

Incentivos para promocionar el aprendizaje

\begin{tabular}{|c|c|}
\hline INCENTIVOS & $\%$ \\
\hline SI & $33 \%$ \\
\hline NO & $67 \%$ \\
\hline TOTAL & $100 \%$ \\
\hline
\end{tabular}

Fuente: Elaboración propia

¿Se cuenta con incentivos para promocionar el aprendizaje organizacional?

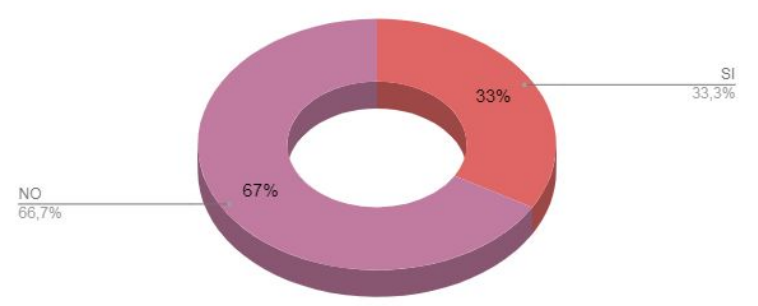

Fuente: Elaboración propia

¿Cuáles son los SI que proporcionen datos sobre el desempeño de la organización y de sus componentes?

Tabla 6.1:

Tipos de SI para el desempeño organizacional 


\begin{tabular}{|c|c|}
\cline { 2 - 2 } INCENTIVOS & $\%$ \\
\hline $\begin{array}{c}\text { Entrenadores } \\
\text { departamentales }\end{array}$ & $\mathbf{5 0 \%}$ \\
\hline $\begin{array}{c}\text { Días otorgados } \\
\text { remunerados por } \\
\text { antigüedad, Dinero, } \\
\text { Bonos. }\end{array}$ & $\mathbf{5 0 \%}$ \\
\hline TOTAL & $\mathbf{1 0 0 \%}$ \\
\hline
\end{tabular}

Fuente: Elaboración

propia

¿Cuáles son los SI que proporcionen datos sobre el desempeño de la organización y de sus compone...

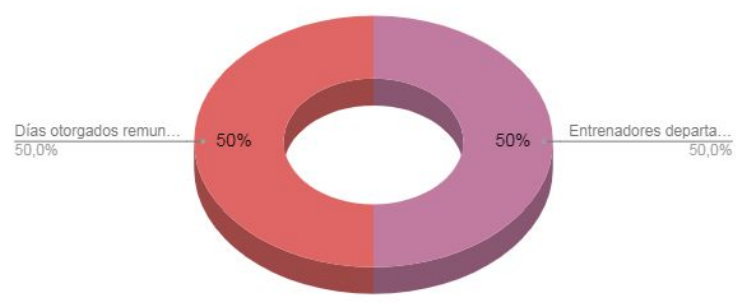

Fuente: Elaboración propia

7. ¿Cuál es la actitud de los miembros de la organización hacia el aprendizaje personal?

Tabla 7:

Tipos de SI para el desempeño organizacional

\begin{tabular}{|c|c|}
\cline { 2 - 2 } ACTITUD PERSONAL & $\%$ \\
\hline Interesada & $\mathbf{1 0 0 \%}$ \\
\hline TOTAL & $\mathbf{1 0 0 \%}$ \\
\hline
\end{tabular}

Fuente: Elaboración propia

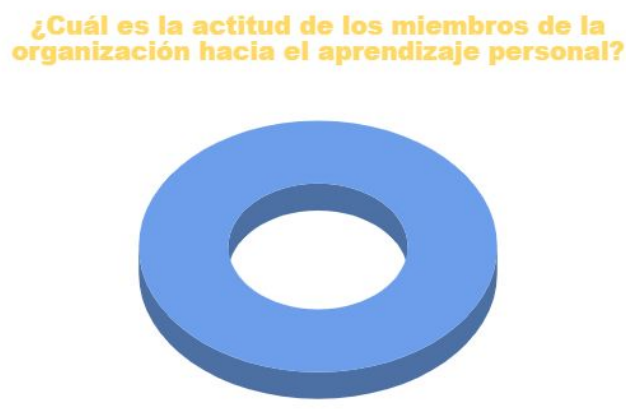

Fuente: Elaboración propia

7.1 ¿Esa actitud facilita el aprendizaje?

\begin{tabular}{|c|c|}
\cline { 2 - 2 } APRENDIZAJE & $\%$ \\
ORGANIZACIONAL & \\
\hline SI & $\mathbf{7 5 \%}$ \\
\hline NO & $\mathbf{2 5 \%}$ \\
\hline TOTAL & $\mathbf{1 0 0 \%}$ \\
\hline
\end{tabular}

Fuente: Elaboración propia

\section{¿Esa actitud facilita el aprendizaje?}

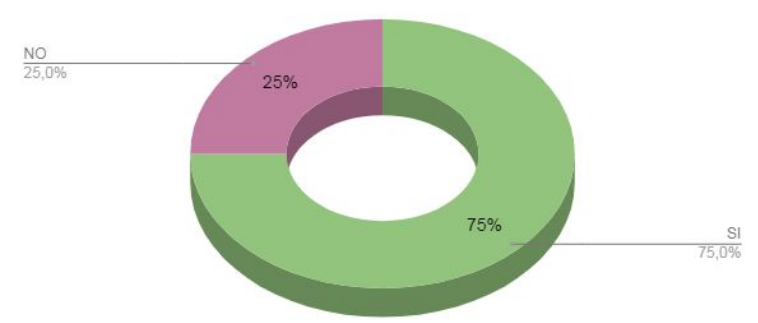

Fuente: Elaboración propia 
7.2 ¿De qué forma facilita el aprendizaje organizacional?

\begin{tabular}{|c|c|}
\hline $\begin{array}{l}\text { APRIENDIZAJE } \\
\text { ORGANIZACIONAL }\end{array}$ & $\%$ \\
\hline $\begin{array}{c}\text { Equipo motivado hacia el } \\
\text { aprendizaje }\end{array}$ & $33 \%$ \\
\hline Opinión colaboradores & $33 \%$ \\
\hline $\begin{array}{l}\text { Colaboración } 0 \\
\text { capacitación a miembros }\end{array}$ & $33 \%$ \\
\hline TOTAL & $100 \%$ \\
\hline
\end{tabular}

Fuente: Elaboración propia

¿De qué forma facilita el aprendizaje organizacional?

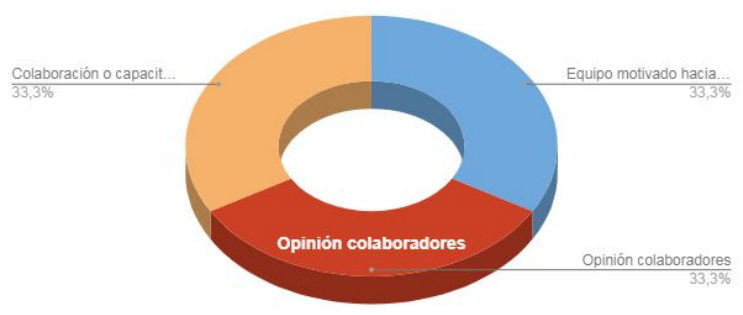

Fuente: Elaboración propia

8. ¿Los líderes de los proceso fomentan el aprendizaje organizacional?

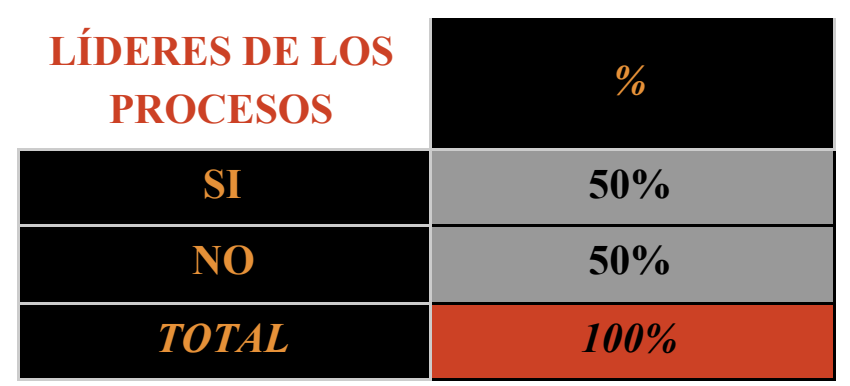

Fuente: Elaboración propia

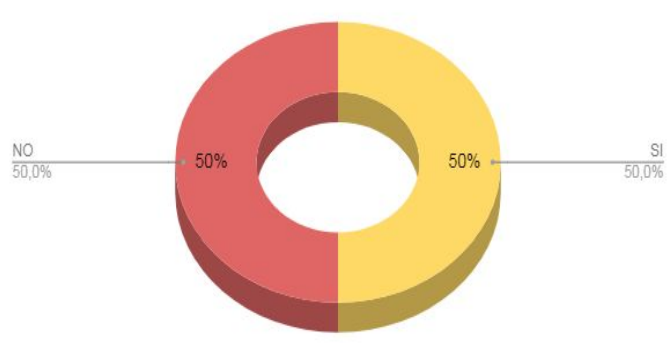

8.1 ¿De qué forma fomentan el aprendizaje organizacional?

\begin{tabular}{|c|c|}
\hline $\begin{array}{l}\text { LÍDERES DE LOS } \\
\text { PROCESOS }\end{array}$ & $\%$ \\
\hline $\begin{array}{l}\text { Liderazgo e iniciativa } \\
\text { en capacitaciones }\end{array}$ & $33 \%$ \\
\hline $\begin{array}{c}\text { Creación de un Plan } \\
\text { Anual }\end{array}$ & $33 \%$ \\
\hline $\begin{array}{c}\text { Capacitaciones } \\
\text { realizadas } 102 \text { veces } \\
\text { al año }\end{array}$ & $33 \%$ \\
\hline TOTAL & $100 \%$ \\
\hline
\end{tabular}

Fuente: Elaboración propia

¿De qué forma fomentan el aprendizaje organizacional?

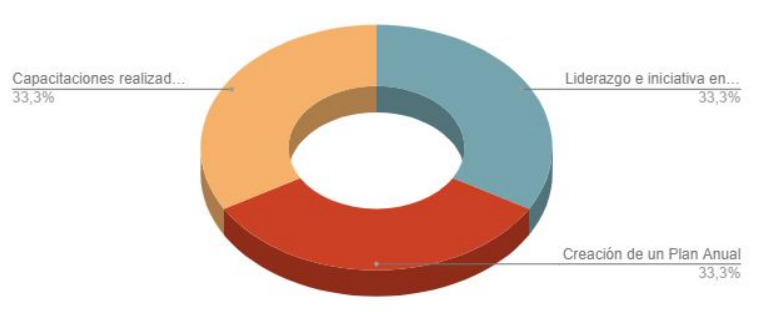

Fuente: Elaboración propia 
9. ¿Los líderes de los proceso fomentan el aprendizaje organizacional?

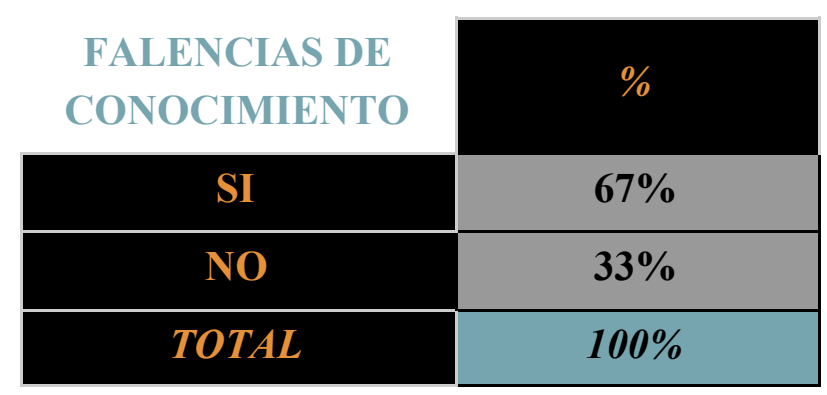

Fuente: Elaboración propia

¿Las personas de la organización pueden reconocer falencias de conocimiento?

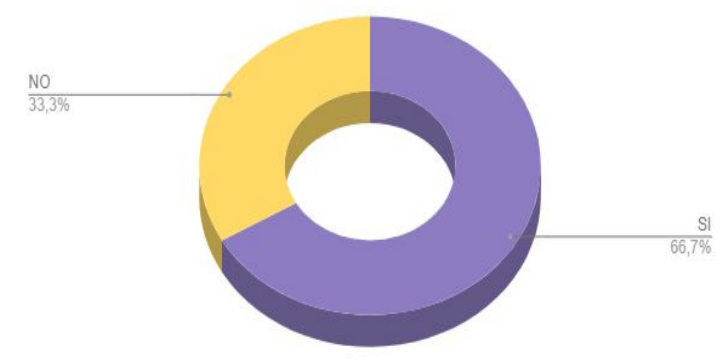

Fuente: Elaboración propia

9.1 ¿Es posible convertirse en estudiantes mostrando la responsabilidad del aprendizaje?

\begin{tabular}{|c|c|}
\hline $\begin{array}{c}\text { RESPONSABILIDAD } \\
\text { APRENDIZAJE }\end{array}$ & $\%$ \\
\hline SI & $100 \%$ \\
\hline NO & $0 \%$ \\
\hline TOTAL & $100 \%$ \\
\hline
\end{tabular}

¿Es posible convertirse en estudiantes mostrando la responsabilidad del aprendizaje?

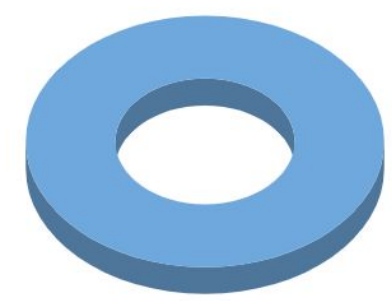

Fuente: Elaboración propia

9.2 ¿Cómo pueden convertirse en estudiantes mostrando la responsabilidad del aprendizaje?

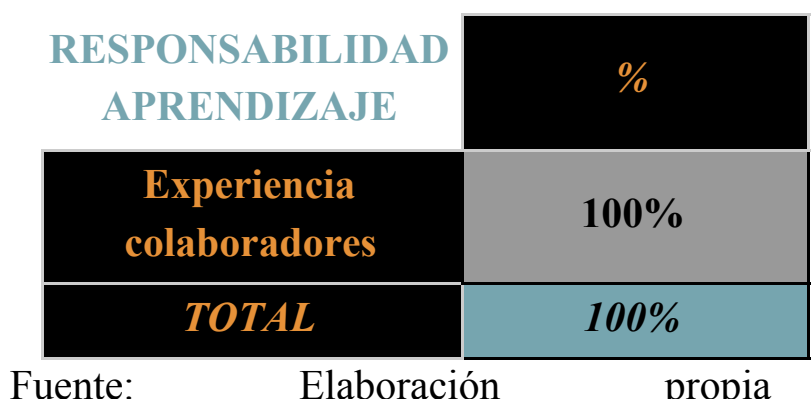

Fuente:

Elaboración

propia

¿Cómo pueden convertirse en estudiantes mostrando la responsabilidad del aprendizaje?

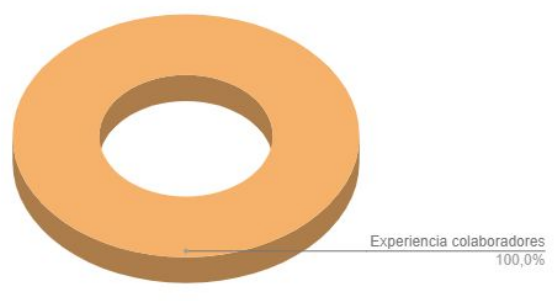

Fuente: Elaboración propia

10. ¿Existe un proceso formal de aprendizaje en la organización? 


\begin{tabular}{|c|c|}
\cline { 2 - 2 } PROCESO DE & $\%$ \\
APRENDIZAJE & \\
\hline SI & $\mathbf{5 0 \%}$ \\
\hline NO & $\mathbf{5 0 \%}$ \\
\hline TOTAL & $100 \%$ \\
\hline
\end{tabular}

Fuente: Elaboración propia

¿Existe un proceso formal de aprendizaje en la organización?

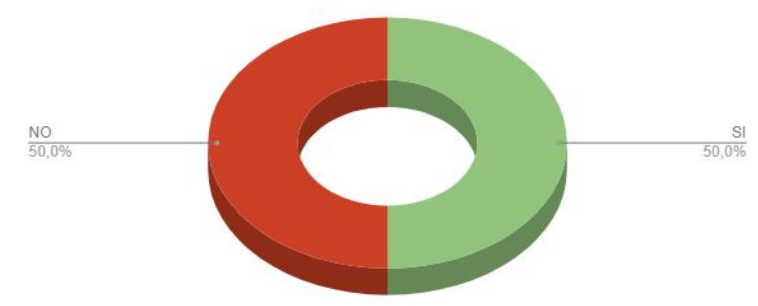

Fuente: Elaboración propia

10.1 ¿El proceso formal de aprendizaje hace parte de la cultura de la organización?

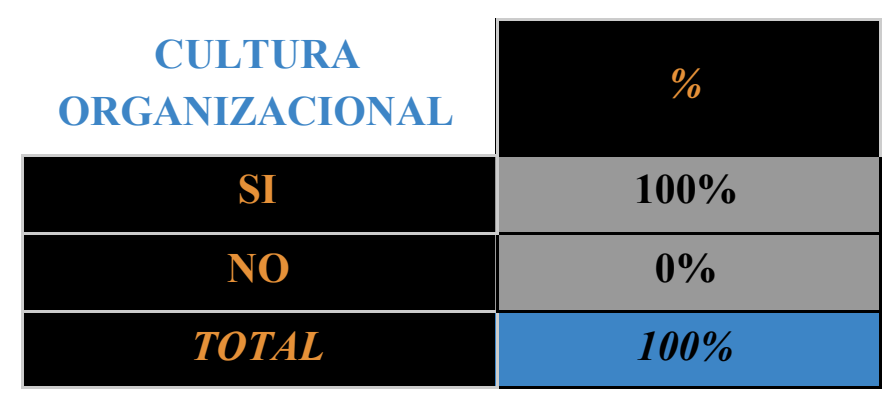

Fuente: Elaboración propia

¿El proceso formal de aprendizaje hace parte de la cultura de la organización?

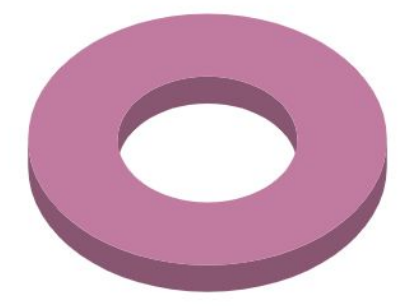

Fuente: Elaboración propia
10.2 ¿El acceso al proceso formal de aprendizaje es voluntario y obligatorio?

\begin{tabular}{|c|c|}
\cline { 2 - 2 } & $\%$ \\
\hline PROCESO FORMAL & \\
\hline VOLUNTARIO & $67 \%$ \\
\hline TOTAL & $33 \%$ \\
\hline
\end{tabular}

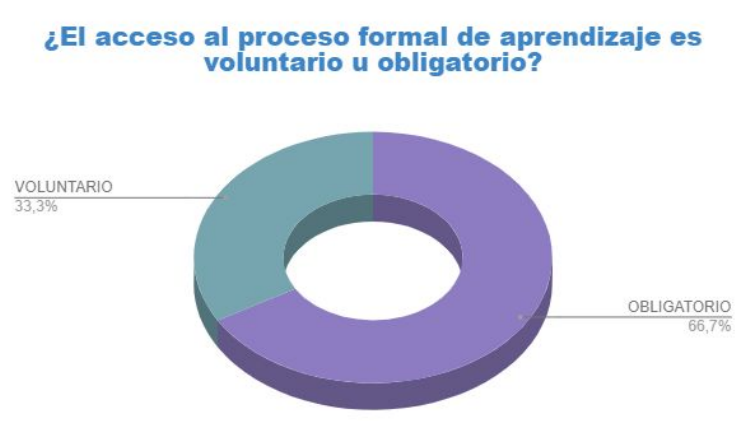

10.3 ¿Cómo se realiza el proceso de aprendizaje en la organización?

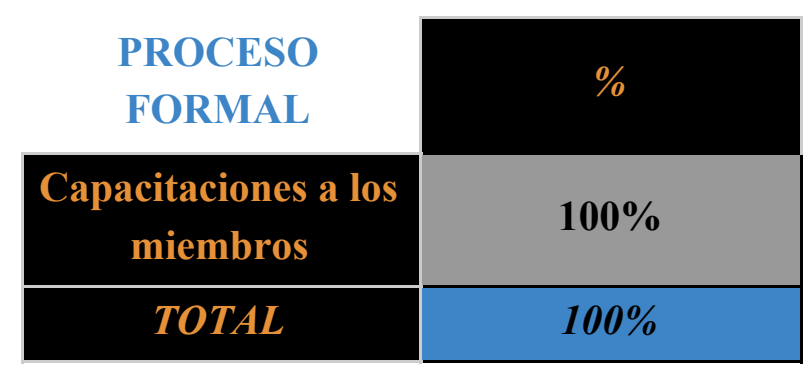

Fuente: Elaboración propia ¿Cómo se realiza el proceso de aprendizaje en la
organización?

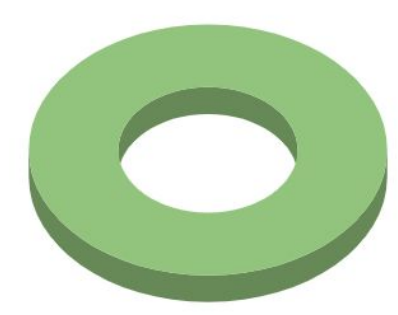

Fuente: Elaboración propia 


\section{BIBLIOGRAFÍA}

- Es.investinbogota.org. (2016). Bogotá concentra la inversión en infraestructura hotelera del país | Invest In Bogotá. [online] Available at: https://es.investinbogota.org/noticias/bogo ta-concentra-la-inversion-en-infraestructur a-hotelera-del-pais [Accessed 17 Mar. 2016].

- Aramburu, N (2000)“Un estudio del aprendizaje organizativo desde la perspectiva del cambio", Universidad de Deusto.

- Argyris C. 2001. Sobre el aprendizaje organizacional. Segunda Edición. OXFORD University Press. México D.F.

- Rodríguez Antón, J.M. (2004): Aprendizaje Organizativo, Documento Intelectus $n^{\circ}$ 7, IADE-CIC, Madrid.

https://yiminshum.com/matriz-evaluacionfactores-internos-mefi/

- Dynamic Manufacturing (1988) de Hayes y Cols, Encontrado en Argiris 2001

- Contreras, J. (2018). Matriz DOFA y PEYEA. Recuperado de http://www.joseacontreras.net/direstr/cap8 2d.htm

- LA quinta disciplina. Peter Senge 1993. Encontrado en Argyris 2001

- Mayo, A., \& Lank, E. (2003). Las Organizaciones que Aprenden (The Power of Learning). Barcelona: Gestión 2000, S.A.

- Riquelme Leiva, M. (2016). FODA: Matriz o Análisis FODA - Una herramienta esencial para el estudio de la empresa. Santiago, Chile. Recuperado de https://www.analisisfoda.com/

- Barthelmess, C. (2003). La planeación estratégica en las organizaciones Estrategias y dirección estratégica.
- Serna, H. (2000). Gerencia estratégica: planeación y gestión, teoría y metodología. Colombia: 3Eeditores. 